\title{
Relación entre ergonomía y trastornos músculo esqueléticos en el área de fabricación de jean’s, caso del Consorcio Jean's Ramos Llerena.
}

\begin{abstract}
Relationship between ergonomics and muscle skeletal disorders in the jean's manufacturing area, case of the jean's Ramos Llerena consortium.
\end{abstract}

Jorge Luis Morales Sánchez. ${ }^{1}$, Manolo Alexander Córdova Suárez. ${ }^{2}$ \& Vladimir Vega Falcón. ${ }^{3}$

\begin{abstract}
. DOI: https://doi.org/10.33262/concienciadigital.v4i1.2.1586

Introduction: A study was carried out to relate ergonomics and musculoskeletal disorders in the jean manufacturing area of the Jean's Ramos Llerena Consortium with exposure to work postures and repetitive movements using the OCRA index, the REBA method and the Questionnaire Nordic Standardized, the study verified the presence of musculoskeletal disorders in the neck and dorsal or lumbar region due to repetitive movements and inappropriate postures, as well as short recovery times. Methods: The research design was analytical, cross-sectional, prospective and descriptive. A sample of 43 workers from the jean manufacturing area was used, an evaluation of the position was carried out with the matrix of occupational risks, application of the OCRA index, REBA method and Standardized Nordic Questionnaire, all these methods previously reviewed and validated for their application with teachers and professionals related to the subject. Results: In the matrix of occupational risks, the presence of repetitive movements and inappropriate postures was detected with a Very Severe risk level, the OCRA method demonstrated an unacceptable level of risk of repetitive movements, the REBA method

\footnotetext{
${ }^{1}$ Regional Autonomous University of the Andes, Faculty of Medical Sciences, Ambato, Tungurahua, pg.jorgelms98@uniandes.edu.ec, orcid: 0000-0003-3335-0480

${ }^{2}$ G+ Energy-Risks \& Engineering Group, Department of Food Science and Engineering, Technical University of Ambato, Ambato, Tungurahua, ma.cordova@uta.edu.ec, orcid: 0000-0001-6786-7926

${ }^{3}$ Regional Autonomous University of the Andes, Faculty of Medical Sciences, Ambato, Tungurahua, ua.vladimirvega@ uniandes.edu.ec, orcid: 0000-0003-0140-4018
} 
demonstrated a final score of 6 , medium risk and a necessary action for inappropriate postures. In the Standardized Nordic Questionnaire, workers perform between 2 or 3 activities with a time of approximately 1 to 5 years in the company, there are discomfort in the neck in 37 workers $(86 \%)$ and in the dorsal or lumbar region in 38 workers $(88$, $3 \%$ ) with absenteeism between 1 to 7 days clearly attributed to work. Conclusion: It was concluded that there is a high relationship between ergonomics and musculoskeletal disorders since most of the workers presented discomfort in the neck and dorsal or lumbar spine due to routine at work, poor postural habits and repetitive movements.

Keywords: Ergonomics, jean manufacturing, REBA, OCRA, musculoskeletal disorders.

\section{Resumen.}

Introducción: Se realizó un estudio para relacionar la ergonomía y los trastornos músculo esquelético en el aérea de fabricación de jean's del Consorcio Jean's Ramos Llerena con exposición a posturas de trabajo y movimientos repetitivos utilizando el índice OCRA, método REBA y el Cuestionario Nórdico Estandarizado, en el estudio se verificó la presencia de trastornos músculo esqueléticos en el cuello y región dorsal o lumbar debido a movimientos repetitivos y posturas inadecuadas, así como también a escasos tiempos de recuperación. Métodos: El diseño de la investigación fue analítico, transversal, prospectivo y descriptivo. Se utilizó una muestra de 43 trabajadores del área de fabricación de jean's, se realizó una evaluación del puesto con la matriz de riesgos laborales, aplicación del índice OCRA, método REBA y Cuestionario Nórdico Estandarizado, todos estos métodos previamente revisados y validados para su aplicación con docentes y profesionales afines al tema. Resultados: En la matriz de riesgos laborales se detectó la presencia de movimientos repetitivos y posturas inadecuadas con un nivel de riesgo Muy Grave, el método OCRA demostró un nivel de riesgo de movimientos repetitivos no aceptable, el método REBA demostró una puntuación final de 6, riesgo medio y una actuación necesaria para posturas inadecuadas. En el Cuestionario Nórdico Estandarizado los trabajadores realizan entre 2 o 3 actividades con un tiempo de aproximadamente 1 a 5 años en la empresa, existen molestias en el cuello en 37 trabajadores $(86 \%)$ y en la región dorsal o lumbar en 38 trabajadores $(88,3 \%)$ con ausentismo entre 1 a 7 días atribuido netamente al trabajo. Conclusión: Se concluyó que existe una alta relación entre ergonomía y los trastornos músculo esqueléticos ya que la mayoría de trabajadores presentaron molestias en el cuello y columna dorsal o lumbar debido a la rutina en el trabajo, los malos hábitos posturales y movimientos repetitivos.

Palabras claves: Ergonomía, fabricación de jean, REBA, OCRA, trastornos músculo esqueléticos.

\section{Introducción.}

La Organización Mundial de la Salud (OMS), establece que "La salud no es sólo la ausencia de enfermedad, sino también es el estado de bienestar somático, psicológico y 
social del individuo y de la colectividad (Navarro, 1998). La importancia de la evaluación continua, y la aplicación de las normas de seguridad ocupacional, por lo que los empleadores son responsables de entregar a sus trabajadores ambientes adecuados, prevenir riesgos y adoptar medidas preventivas, que eviten sobrecargas laborales, exposición a agentes biológicos, físicos y químicos peligrosos. Se trabaja durante 8 horas y a veces más, es mayor el tiempo de labores que el tiempo familiar y de esparcimiento; se debe cuidar que las condiciones de trabajo no sean estresantes. (SICE, 2019). Es importante señalar que Ecuador, desde su constitución respalda el derecho al trabajo en condiciones óptimas. "Toda persona tendrá derecho a desarrollar sus labores en un ambiente adecuado y propicio, que garantice su salud, integridad, seguridad, higiene y bienestar". (Constitución del Ecuador, 2019).

El Instituto Ecuatoriano de Seguridad Social (IESS) y el Ministerio de Salud Pública (MSP), en articulación con el Ministerio del Trabajo (MDT) realizan investigaciones sobre la prevención de riesgos y medio ambiente laboral. Está legislado el reglamento de seguridad y salud de los trabajadores, así como el mejoramiento del medio ambiente de trabajo (Ministerio del Trabajo del Ecuador, 2019).

Por otra parte, existen múltiples clasificaciones de riesgos laborales, por lo que los trastornos musculo esqueléticos están estrechamente relacionados a malas posturas o movimientos repetitivos que los trabajadores realizan en sus actividades y es por esto que dichos trastornos son los problemas más importantes de salud en el trabajo en países industrializados y en vías de desarrollo. En países nórdicos se calcula un gasto de 2.7 y $5.2 \%$ del producto interno bruto. Aproximadamente los problemas músculo-esqueléticos son atribuibles al trabajo en un $30 \%$, por lo que su prevención sería muy aceptable. Los costos económicos por trastornos músculo esqueléticos, en días perdidos de trabajo e invalidez alcanzan a 215 mil millones de dólares al año en Estados Unidos. Para la Agencia Europea para la Seguridad y Salud en el Trabajo, los trastornos músculo esqueléticos afectan a una cuarta parte de la población europea (25\% de los trabajadores sufren dolor de espalda y $23 \%$ dolores musculares). La VI Encuesta Nacional de Condiciones de Trabajo (INSHT) muestra que 74.2\% de los trabajadores sienten alguna molestia músculo esquelética atribuida a posturas y esfuerzos derivados del trabajo específicamente en las de la zona baja de la espalda, nuca-cuello y la zona alta de la espalda son las más frecuentes $(40.1,27$, y 26.6\%, respectivamente). Los trastornos músculo esqueléticos son la principal causa de ausentismo laboral en todos los países miembros de la Unión Europea, reducen la rentabilidad de las empresas y aumentan los costos sociales públicos. (Arenas-Ortiz, 2018).

En Ecuador según el IESS, se registraron aproximadamente 14000 enfermedades ocupacionales, pero menos del $3 \%$ fueron reportadas. Las enfermedades ocupacionales que se presentan con mayor frecuencia en el Ecuador son: el codo de tenista, dolor en la base del talón, dolor de cuello, síndrome túnel del carpo, lumbalgia y dolor e hinchazón en la base del pulgar. (Puente Avila, M.E, 2017).

En la provincia de Tungurahua se encuentra el cantón Pelileo conocida como la ciudad Azul gracias a la producción de prendas en jean's, ésta actividad representa el $42 \%$ de la actividad económica del cantón (Ecuale, 2015), anteriormente no se han encontrado estudios relacionados con ergonomía y trastornos músculo esqueléticos en trabajadores 
de jean's por lo que éste trabajo tiene una importancia significativa por que servirá de línea base para el diseño de puestos ergonómicos en el área de fabricación de jean que contemple trastornos músculo esqueléticos.

En este contexto el objetivo del estudio fue relacionar la ergonomía y los trastornos músculo esquelético en el aérea de fabricación de jean's del Consorcio Jean's Ramos Llerena.

\section{Materiales y métodos}

El estudio afronta la realidad con el problema detectado en el Consorcio Jean's Ramos Llerena, aplicando el método científico (Gómez, y otros, 2017).

Para el inicio de la investigación se realizó una evaluación del puesto con matriz de riesgos laborales en donde se observó afectación por movimientos repetitivos y posturas de trabajo inadecuadas, se realizó un estudio analítico, transversal, prospectivo y descriptivo, desde enero a septiembre del 2020 a 43 trabajadores del área de fabricación de jean's, se utilizó el índice OCRA para medir el nivel de riesgo en función de la probabilidad de aparición de trastornos músculo-esqueléticos en un determinado tiempo, centrándose en la valoración del riesgo en los miembros superiores del cuerpo ya que se considera en la valoración los factores de riesgo recomendados por la IEA (International Ergonomics Association): repetitividad, posturas inadecuadas o estáticas, fuerzas, movimientos forzados y la falta de descansos o periodos de recuperación, valorándolos a lo largo del tiempo de actividad del trabajador, se consideran otros factores influyentes como las vibraciones, la exposición al frío o los ritmos de trabajo. Por ello, existe consenso internacional en emplear el método OCRA para la valoración del riesgo por trabajo repetitivo en los miembros superiores, y su uso es recomendado en las normas ISO 11228-3 y EN 1005-5. (Picazo, A. R., 2016).

El Check List OCRA realiza un detallado análisis de los factores de riesgo relacionados con el puesto de trabajo. Para obtener este nivel de riesgo se analizan los diferentes factores de riesgo de forma independiente, ponderando su valoración por el tiempo durante el cual cada factor de riesgo está presente dentro del tiempo total de la tarea. De esta forma se puntúan los diferentes factores de riesgo, empleando escalas que pueden ser distintas para cada uno. A partir de los valores de las puntuaciones de cada factor se obtiene el Índice Check List OCRA, valor numérico que permite clasificar el riesgo como Aceptable (hasta 7,5), Muy Ligero (7,6 a 11), Ligero (11,1 a 14), Medio (14,1 a 22,5) o Alto (+22,5). A partir de esta clasificación del riesgo, se sugieren acciones correctivas como llevar a cabo mejoras del puesto, la necesidad de supervisión médica o el entrenamiento específico de los trabajadores para ocupar el puesto. (Diego-Mas, 2015).

Por otra parte, se utilizó el método REBA que fue propuesto por Sue Hignett y Lynn McAtamne en el año 2000, esta herramienta ergonómica resultó del trabajo de un grupo de ergonómicos, fisioterapeutas, terapistas ocupacionales y enfermeras que identificaron 600 posturas y con el objetivo de evaluar las condiciones de trabajo y la carga postural, para estimar el riesgo de padecer desórdenes corporales relacionados con el trabajo, y 
evitar las posibles lesiones posturales (Cuixart, 2016). En la actualidad, la aplicación del método no solo es usada como evaluación ergonómica del personal sanitario, si no también aplicable en empleados del sector industrial ya que es considerada como un instrumento capaz de evaluar el nivel de riesgo de presentar trastornos musculo esqueléticos previniendo al evaluador de una posible condición de trabajo inadecuada y la urgencia con la que se debería aplicar acciones correctivas en el puesto de trabajo. (Schwartz y otros, 2019)

Además, se encuestó por vía online con el Cuestionario Nórdico Estandarizado (google form) el cual es usado para la detección y análisis de síntomas musculo esqueléticos, aplicable en el contexto de estudios ergonómicos o de salud ocupacional con el fin de detectar la existencia de síntomas iniciales, que todavía no han constituido enfermedad o no han llevado aún a consultar al médico. Aunque no podemos olvidar que la exploración física sigue siendo esencial para la valoración del diagnóstico clínico, el uso de este cuestionario se ha consagrado como un gran aliado en la detección y estudio de los trastornos musculo esqueléticos en el medio laboral ya que permite detectar la existencia de síntomas iniciales, otorgando un diagnóstico precoz. Las preguntas se concentran en la mayoría de los síntomas que frecuentemente se detectan en diferentes actividades laborales. (Jarreta, 2014)

\section{Resultados.}

Luego de seleccionar los puestos de trabajo, se realizó la matriz de riesgos laborales en el cual se obtuvo los siguientes resultados como se detalla a continuación:

Tabla 1. Matriz de Riesgos Laborales*

\begin{tabular}{ccccc}
\hline RIESGO & $\begin{array}{c}\text { Probabilidad } \\
\text { (Ocurrencia) }\end{array}$ & $\begin{array}{c}\text { Gravedad } \\
\text { (Impacto) }\end{array}$ & $\begin{array}{c}\text { Valor del } \\
\text { Riesgo }\end{array}$ & $\begin{array}{c}\text { Nivel de } \\
\text { Riesgo }\end{array}$ \\
$\begin{array}{c}\text { Movimientos } \\
\text { repetitivos } \\
\begin{array}{c}\text { Posiciones } \\
\text { inadecuadas }\end{array}\end{array}$ & 4 & 4 & 16 & Muy grave \\
Carga de peso & 2 & 4 & 16 & Muy grave \\
Vibraciones & 1 & 2 & 4 & Apreciable \\
Ruido & 2 & 1 & 1 & Marginal \\
Iluminación & 1 & 1 & 4 & Apreciable \\
\end{tabular}




\section{Superficies \\ irregulares}

Caídas de personas al mismo nivel

Riesgo Químico

Manejo de

herramientas corto

punzantes

\section{1}

2

1

2

1

4

1

$$
1
$$

2
1

Marginal

\begin{abstract}
*Nota: Se realizó la evaluación del puesto de cerrado y empretinado de pantalón por ser el área que más trabajadores realizaban dicha actividad. Interpretación: Riesgo muy grave (15-25): Requiere medidas preventivas urgentes. No se debe iniciar el proyecto sin la aplicación de medidas preventivas urgentes y sin acotar sólidamente el riesgo. Riesgo importante (9-14): Medidas preventivas obligatorias. Se deben controlar fuertemente las variables de riesgo durante el proyecto. Riesgo apreciable (3-8): Estudiar económicamente si es posible introducir medidas preventivas para reducir el nivel de riesgo. Si no fuera posible, mantener las variables controladas. Riesgo marginal (1-2): Se vigilará, aunque no requiere medidas preventivas de partida. Fuente: elaboración propia
\end{abstract}

En la tabla 2 se presenta los datos del método OCRA

Tabla 2. Resultados finales de la evaluación del puesto de trabajo.

Factores de riesgo por trabajo repetitivo

\section{Indicadores \\ Derecha}

Tiempo de recuperación insuficiente

Frecuencia de movimientos

Aplicación de fuerza

Hombro

Codo

Muñeca

Mano-dedos

Estereotipo

Posturas forzadas

Factores de riesgo complementario

Factor Duración

Resultado índice de riesgo

Interpretación
10

Izquierda

$\begin{array}{cc}10 & 10 \\ 3 & 3 \\ 2 & 2 \\ 6 & 6 \\ 2 & 2 \\ 0 & 0 \\ 2 & 2 \\ 1,5 & 1,5 \\ 7,5 & 7,5 \\ 0 & 0 \\ 0,95 & 0,95 \\ 21,38 & 21,38\end{array}$

No aceptable. Nivel medio

Nota: Los valores generados presentes en la tabla se tomaron en condiciones máximas de trabajo en el mismo turno y horario a un promedio de 9 trabajadores del puesto de cerrado y empretinado de pantalón. Fuente: elaboración propia

En la tabla 3 se presentan los datos del método REBA 
Tabla 3. Resultados finales de evaluación del puesto de trabajo

\begin{tabular}{|c|c|c|c|c|c|}
\hline \multicolumn{2}{|c|}{ GRUPO A } & \multicolumn{2}{|c|}{ GRUPO B } & \multicolumn{2}{|c|}{$\begin{array}{c}\text { NIVELES DE } \\
\text { RIESGO Y ACCION }\end{array}$} \\
\hline Área & Puntuación & Área & Puntuación & Indicador & Puntuación \\
\hline Cuello & 2 & Antebrazos & 1 & $\begin{array}{l}\text { Puntuación } \\
\text { final } \\
\text { REBA }\end{array}$ & 6 \\
\hline Piernas & 1 & Muñecas & 1 & $\begin{array}{l}\text { Nivel de } \\
\text { acción }\end{array}$ & 2 \\
\hline Tronco & 4 & Brazos & 2 & $\begin{array}{l}\text { Nivel de } \\
\text { riesgo }\end{array}$ & Medio \\
\hline Carga/Fuerza & 0 & Agarre & 1 & Actuación & $\begin{array}{c}\text { Es } \\
\text { necesario la } \\
\text { actuación }\end{array}$ \\
\hline
\end{tabular}

\section{Resultado de aplicación del cuestionario nórdico estandarizado.}

En el gráfico 1 se presenta la edad de los trabajadores del Consorcio Jean’s RamosLlerena

\section{Gráfico 1: Edad}

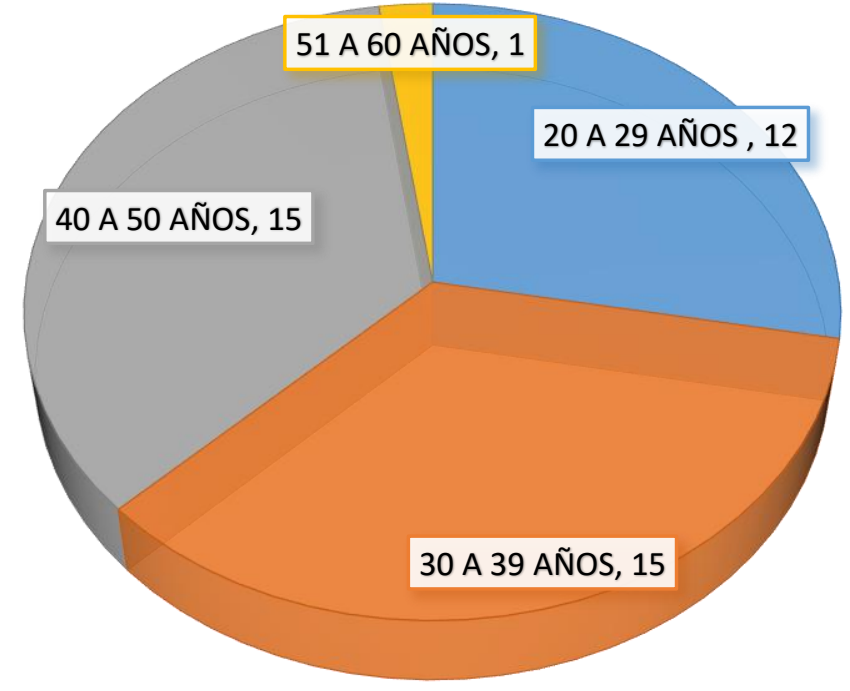

Fuente: elaboración propia

En el gráfico 2 se presenta el puesto de trabajo de los trabajadores del Consorcio Jean's Ramos Llerena 
Gráfico 2: Puesto de trabajo*

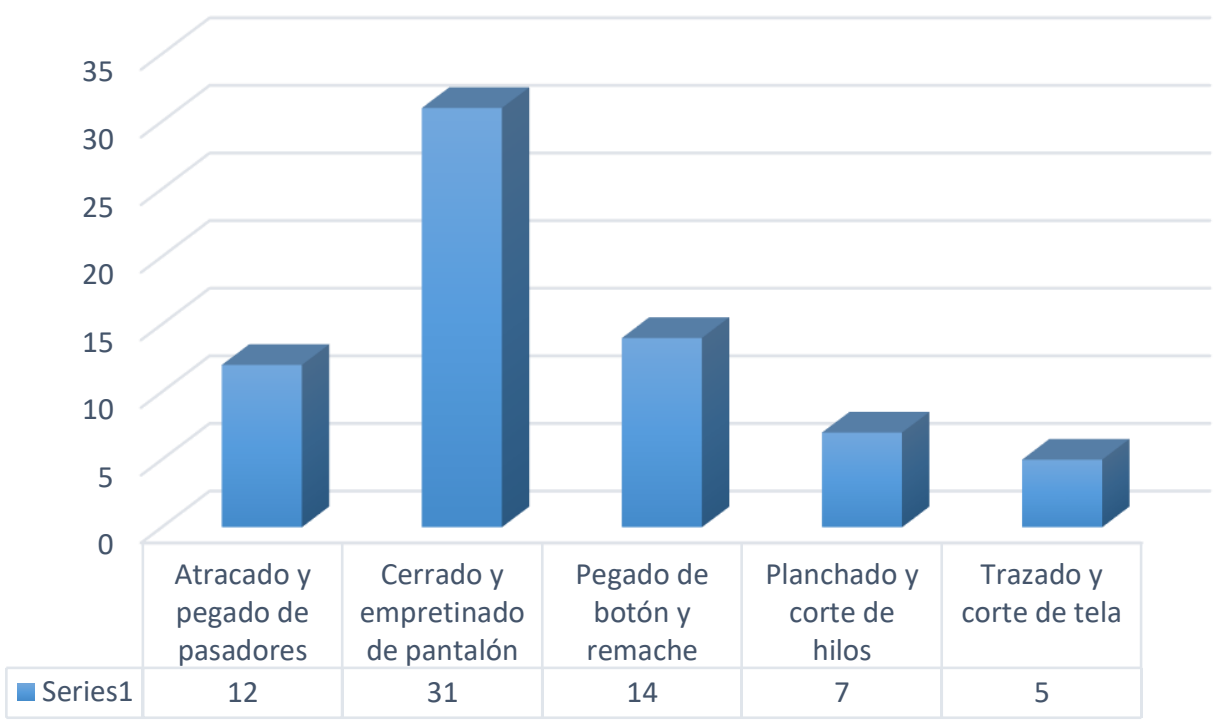

*Nota: indicar que algunos trabajadores realizan 2 o 3 actividades en la misma área de trabajo. Fuente: elaboración propia.

En el gráfico 3 se presenta el tiempo de trabajo de los trabajadores en el Consorcio Jean's Ramos Llerena.

Gráfico 3: Tiempo de trabajo

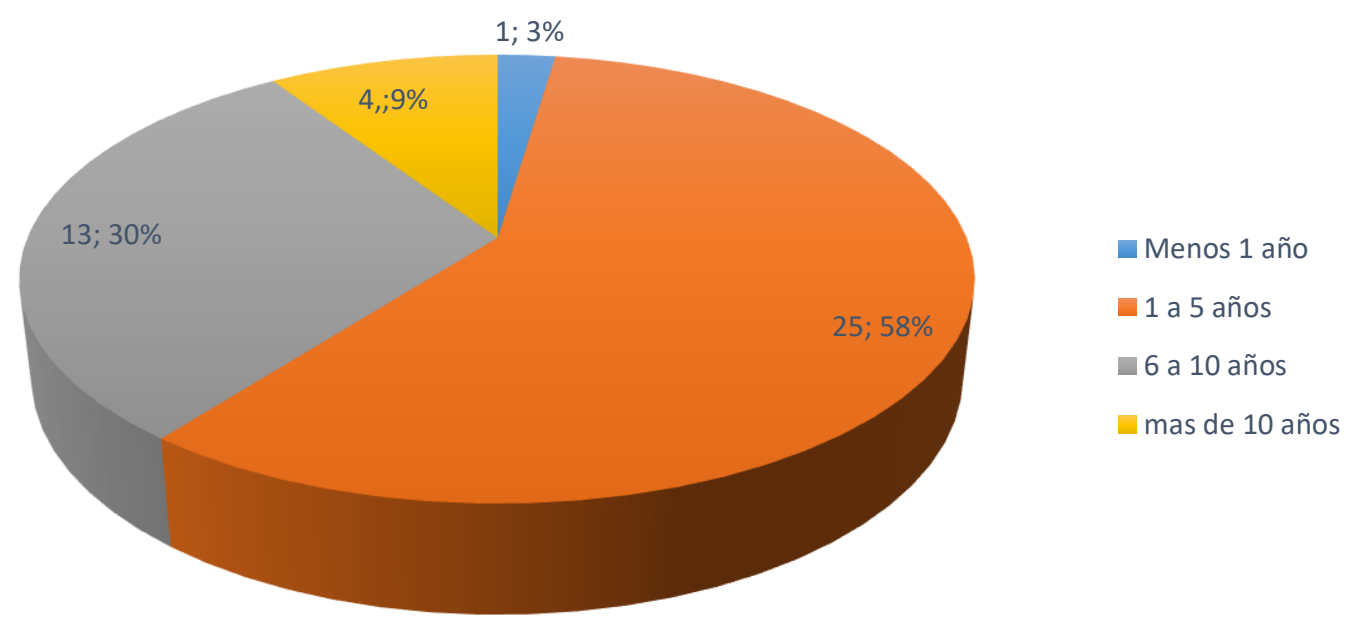

Fuente: elaboración propia

En el gráfico 4 se presenta las molestias encontradas en los trabajadores del Consorcio Ramos Llerena 
Gráfico 4: Molestias de los trabajadores.

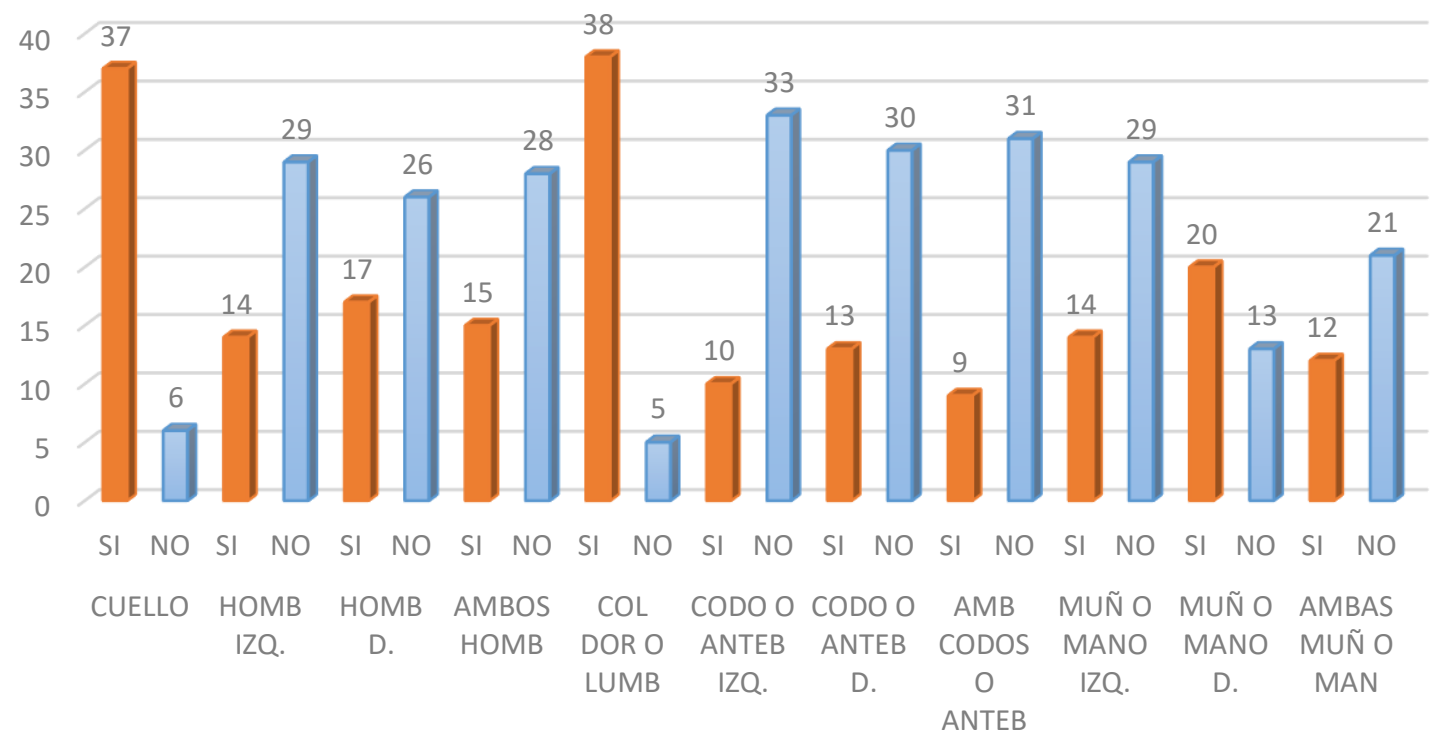

Fuente: elaboración propia.

En el gráfico 5 se presenta las molestias que han presentado los trabajadores del Consorcio Jean's Ramos Llerena en los últimos 12 meses según la región afectada.

Gráfico 5: Molestias en los 12 meses últimos.

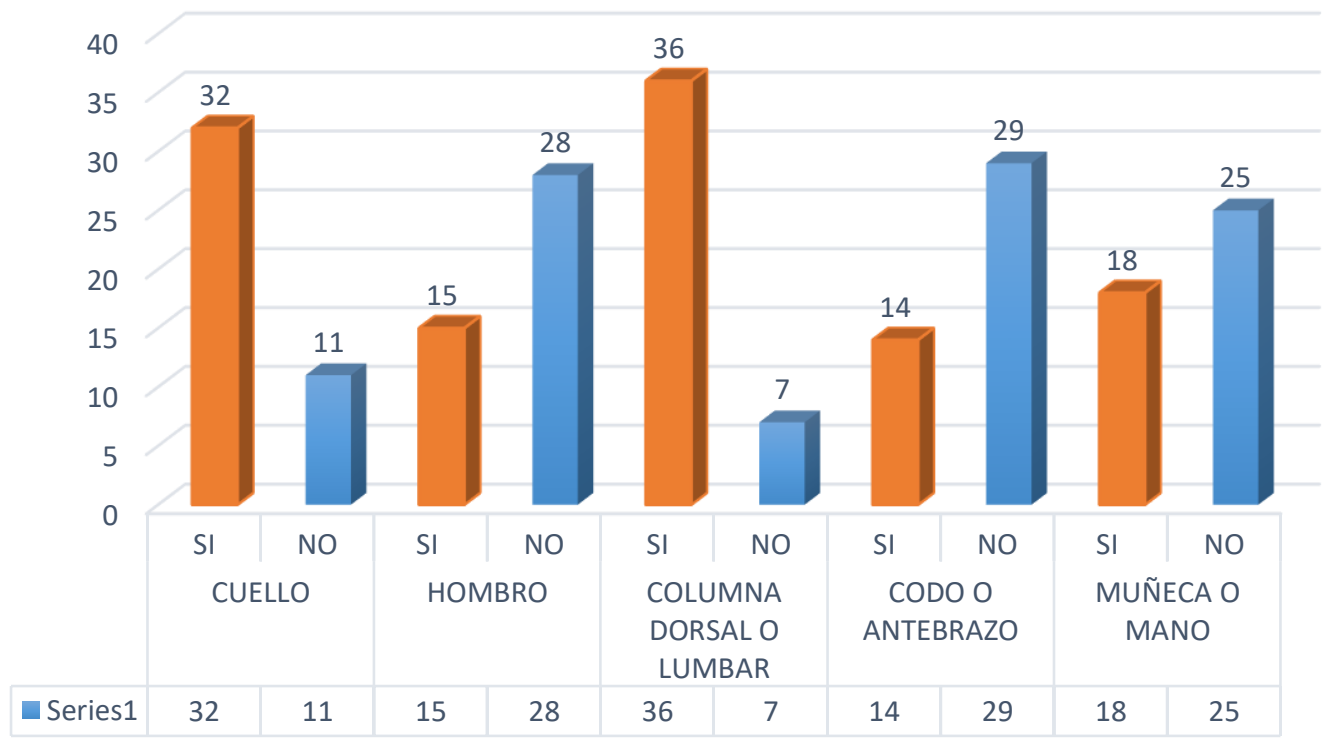

Fuente: elaboración propia.

En el gráfico 6 se presenta el tiempo de ausentismo de los trabajadores del Consorcio Jean’s Ramos Llerena por las diferentes molestias presentadas. 
Grafico 6: Tiempo de ausentismo del lugar de trabajo

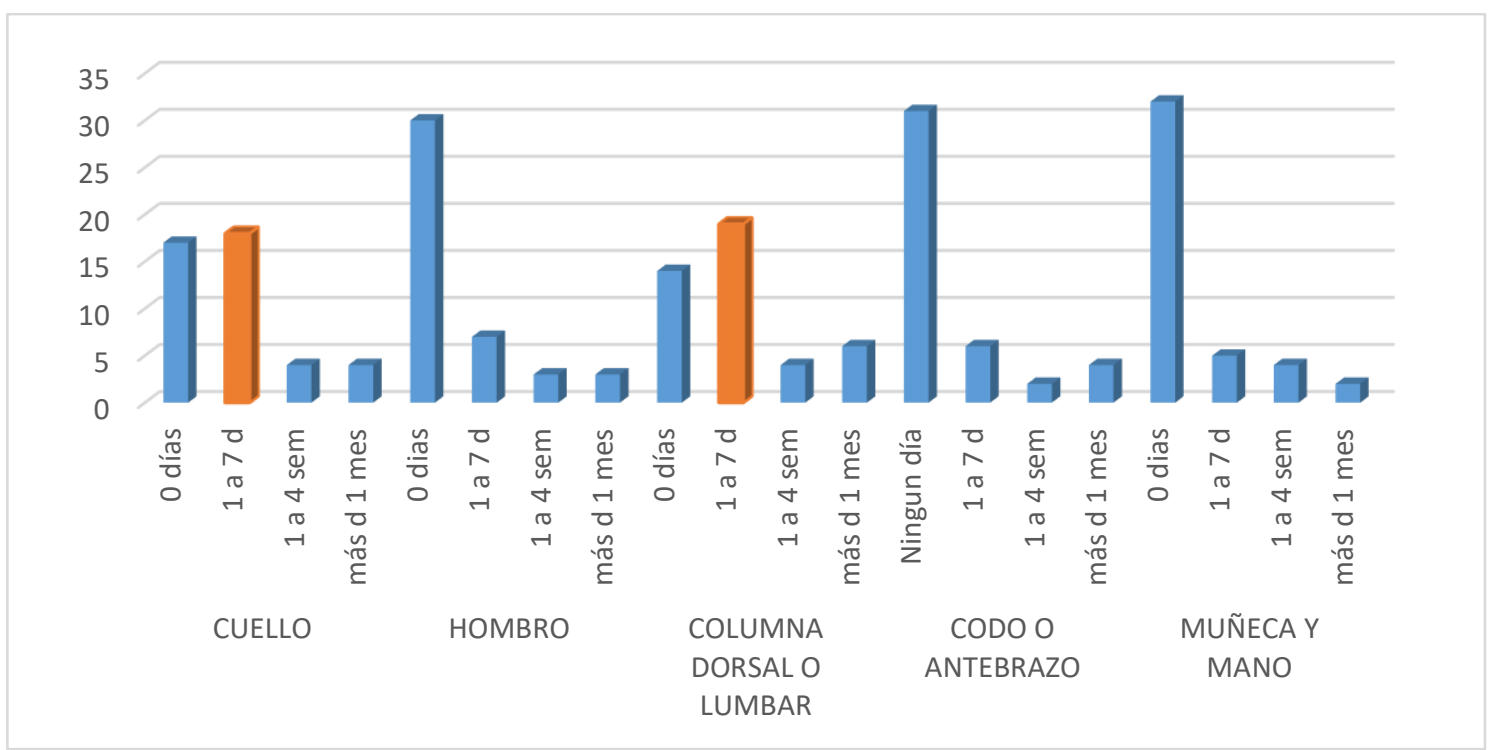

Fuente: elaboración propia.

En el gráfico 7 se presentan las atribuciones de la presencia de las molestias de los trabajadores del Consorcio Jean's Ramos Llerena.

Gráfico 7: Atribuciones de las molestias.

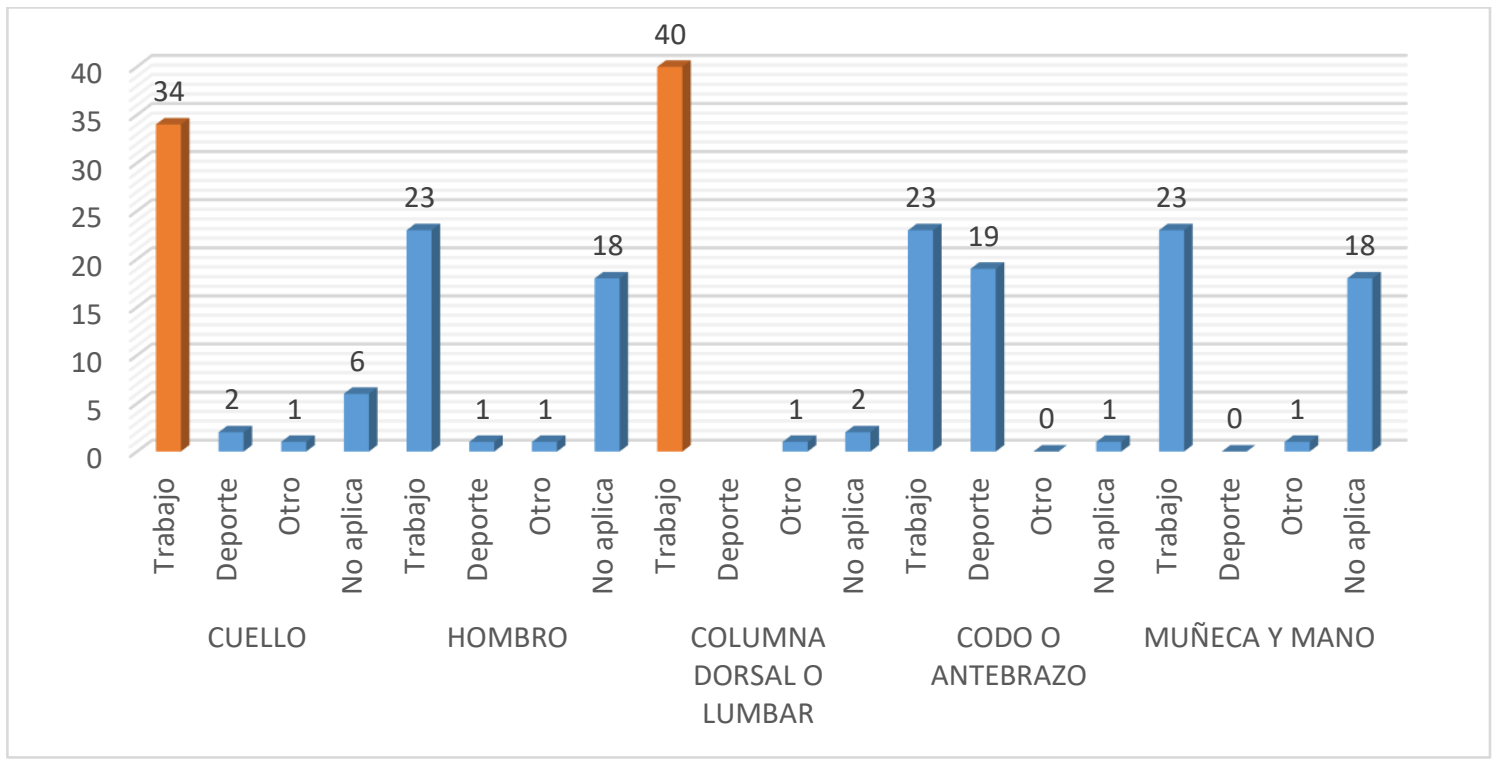

Fuente: elaboración propia

\section{Discusión}

Según lo mostrado en la tabla 1 se puede evidenciar que para el puesto seleccionado para este estudio los valores de movimientos repetitivos y posturas inadecuadas alcanzan un 
nivel de riesgo muy grave (valor: 16) en relación a los demás riesgos que oscilan entre valores marginales (valor 1) y apreciables (valor 4).

En relación a la tabla 2 podemos observar la aplicación del método OCRA en el que los resultados finales del mismo y como evaluación del puesto de cerrado y empretinado de pantalón se obtuvieron tanto para el brazo derecho como para el brazo izquierdo un índice de riesgo de 21,38 dándonos así un valor no aceptable de nivel medio para movimientos repetitivos.

En concordancia con la tabla 3 se muestran los resultados finales de la aplicación del método REBA para el puesto de trabajo seleccionado en donde se observa una puntuación de 6 con un nivel de riesgo medio para posturas inadecuadas y nos indica que es necesario actuar en dicho puesto.

Según el cuestionario nórdico aplicado en la entidad objeto de estudio cuenta con trabajadores en su mayoría de edades comprendidas entre 20 y 50 años como se observa en el gráfico 1. Dentro de éstas edades podemos ver en el gráfico 2 que en mayor proporción existen trabajadores que realizan el proceso de cerrado y empretinado de pantalón (45\%; 31 trabajadores), seguido por pegado de botón y remache $(20 \% ; 14$ trabajadores), atracado y pegado de pasadores (18\%; 12 trabajadores), planchado y corte de hilos (10\%; 7 trabajadores) y por último y con un porcentaje bajo el proceso de trazado y corte de tela ( $7 \% ; 5$ trabajadores), hay que mencionar que algunos trabajadores realizan entre 2 o 3 actividades de las antes mencionadas.

Es importante mencionar que en el gráfico 3 la mayor parte de los trabajadores se encuentran laborando de 1 a 5 años (58\%; 25 trabajadores), seguido de 6 a 10 años (30\%, 13 trabajadores), esto indica que al encontrarse por muchos años dentro de su área de trabajo, es un predictor independiente de trastornos músculo esqueléticos, por lo que de manera relevante se ha dado un gran número de molestias de cuello (37 trabajadores) y columna dorsal o lumbar (38 trabajadores) como se muestra en el gráfico 4, sin embargo, en este estudio existió una relación estadísticamente significativa con los demás trastornos músculo esqueléticos y en menor proporción como por ejemplo molestias en la muñeca derecha (20 trabajadores) o molestias en el hombro derecho (17 trabajadores).

En relación al gráfico 5 el trastorno músculo esquelético más frecuentemente encontrado presente en los últimos 12 meses, fue en la columna dorsal o lumbar presentes en 36 trabajadores, seguido por molestias en el cuello con 32 trabajadores, por lo que los puestos de trabajo juegan un rol importante en el aparecimiento de estas patologías ya que también tiene mucha relación con el ausentismo laboral como nos indica en el gráfico 6 entre 1 a 7 días debido a éstos problemas en 19 trabajadores con molestias en la columna dorsal o lumbar y 18 trabajadores con molestias en el cuello respectivamente, atribuyendo todo esto al trabajo como se muestra en el gráfico 7 con 40 trabajadores para columna dorsal o lumbar y 34 trabajadores para cuello siendo directamente relacionado los trastornos músculo esqueléticos con la ergonomía. 
Para futuras investigaciones se podría considerar poblaciones de trabajadores de jean's más grandes y pertenecientes a otras instituciones, durante un lapso de tiempo mayor para poder fortalecer y, en algunos casos, clarificar las estadísticas documentadas en el presente estudio, además la presente investigación ha sido realizada por primera vez en esta población y puede ser útil como una línea de base para dar un seguimiento posterior. De este modo, a través de este estudio podemos plantear la necesidad de tomar medidas preventivas a corto, mediano y largo plazo, en los trabajadores del área de fabricación de jean's y extrapolarlo a otras poblaciones del mismo sector, para evitar la aparición de trastornos músculo esqueléticos en los trabajadores sanos y en los futuros trabajadores, y evitar la tendencia a la cronicidad de los casos agudos y subagudos.

\section{Conclusiones.}

- En el presente estudio y en el análisis de los resultados se concluye que existe una alta relación entre ergonomía y los trastornos músculo esqueléticos ya que la mayoría de trabajadores presentaron molestias en el cuello (37 trabajadores) y columna dorsal o lumbar (38 trabajadores) debido a la rutina en el trabajo y con 1 a 5 años de la mayoría de trabajadores dentro de la empresa, los malos hábitos posturales y movimientos repetitivos con un nivel de riesgo muy grave según la matriz de riesgos laborales, todo esto asociado a escasos tiempos de recuperación en el área de trabajo.

- Bajo los antecedentes antes mencionados se puede mejorar las condiciones de trabajo al recomendar que se mantenga los pies apoyados por completo en el suelo, las rodillas deben mantener el mismo nivel o estar por encima de las caderas, el respaldo de la silla tiene que ser acorde a las curvaturas fisiológicas de la columna, además tomar una postura recta a la altura de su silla y mesa para ayudar a conseguir la posición de cabeza, cuello, hombro y espalda, así disminuir un alto porcentaje en las molestias de cuello, columna dorsal o lumbar.

- En este contexto se recomienda, además, generar algunas soluciones a los trastornos musculo esqueléticos actuales y evitar su aparición o progresión a futuro, a través de la prevención y mejoramiento de las condiciones de trabajo, así como la capacitación sobre la adecuada ergonomía en el lugar de trabajo.

\section{Referencias bibliográficas.}

Arenas-Ortiz, L., \& Cantú-Gómez, Ó. (2018). Factores de riesgo de trastornos músculoesqueléticos crónicos laborales. Medicina Interna de México, 29(4), 370-379.

Constitución del Ecuador. (26 de junio de 2019). Constitución de la República del Ecuador. Recuperado de https://www.wipo.int/...ocs/laws/es/ec/ec030es.pdf

Cuixart, S. N. (2016). NTP 601: Evaluación de las condiciones de trabajo: carga postural. Método REBA (Rapid Entire Body Assessment). Inst. Nac. Segur. e Hig. en el Trab, 7. 
Diego-Mas, José Antonio. Evaluación del riesgo por movimientos repetitivos mediante el Check List Ocra. Ergonautas, Universidad Politécnica de Valencia, 2015. [consulta 15-10-2020]. Disponible online: http://www.ergonautas.upv.es/metodos/ocra/ocra-ayuda.php

García, A. M., Sevilla, M. J., Gadea, R., \& Casañ, C. (2012). Intervención de ergonomía participativa en una empresa del sector químico. Gaseta Sanitaria-Jurnal Elsevier, 383-386. Recuperado el 13 de julio de 2019, de http://scielo.isciii.es/...s/v26n4/nota_campo3.pdf

Gómez, C., Álvarez, G., Fernández, A., Castro, F., Vega, V., Comas, R., \& Ricardo, M. (2017). La investigación científica y las formas de titulación. Aspectos conceptuales y prácticos. Quito: Editorial Jurídica del Ecuador.

Jarreta, B. M. (2014). Validación del cuestionario nórdico músculo esquelético estandarizado en población española.

ISO. (20 de junio de 2019). ISO Tools. Obtenido de https://www.isotools.org/normas/ I. Kuorinka, B. Jonsson, A. Kilbom, H. Vinterberg, F. Biering-Sørensen, G. Andersson, K. Jørgensen. Standardised Nordic questionnaires for the analysis of musculoskeletal symptoms. Applied Ergonomics 2014, 18.3,233-237

Ministerio del Trabajo del Ecuador. (21 de junio de 2019). Reglamento de Seguridad y Salud de los trabajadores y mejoramiento del medio ambiente de trabajo. Obtenido de http://www.trabajo.gob.ec/...to-Ejecutivo-2393.pdf

Moreno, M. V. (2016). Ergonomía en la práctica odontológica. Revisión de literatura. Revista Venezolana de Investigación Odontológica 106-117, 106-117.

Navarro, V. (1998). Concepto actual de la salud pública. Martínez, F., Castellanos, PL, Navarro, V., Salud Pública, 49-54.

Puente Avila, M. E. (2017). Propuesta de un estudio ergonómico para prevención de trastornos músculo-esqueléticos y enfermedades laborales en el personal de producción, empaque y bodega de una empresa farmacéutica en el primer semestre 2018.

Picazo, A. R., \& de Miguel, J. L. (2016). NTP 629: Movimientos repetitivos: métodos de evaluación Método OCRA: actualización.

SICE. (21 de junio de 2019). Sistema de información sobre comercio exterior. OEA. Recuperado de http://www.sice.oas.org/.../Decisiones/DEC584s.asp

Schwartz, AH, Albin, TJ y Gerberich, SG (2019). Fiabilidad intra e interevaluador de la herramienta de evaluación rápida de cuerpo entero (REBA). Revista Internacional de Ergonomía Industrial, 71, 111-116.

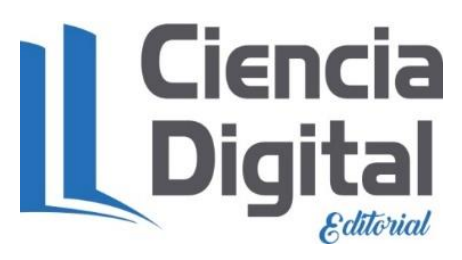




\section{PARA CITAR EL ARTÍCULO INDEXADO.}

Córdova Suárez, M. A., Morales Sánchez, J. L., \& Vega Falcón, V. (2021). Relación entre ergonomía y trastornos músculo esqueléticos en el área de fabricación de jean's, caso del Consorcio Jean's Ramos Llerena. ConcienciaDigital, 4(1.2), 162-175. https://doi.org/10.33262/concienciadigital.v4i1.2.1586

\section{Ciencia \\ LDigital}

El artículo que se publica es de exclusiva responsabilidad de los autores y no necesariamente reflejan el pensamiento de la Revista Conciencia Digital.

El artículo queda en propiedad de la revista y, por tanto, su publicación parcial y/o total en otro medio tiene que ser autorizado por el director de la Revista Conciencia Digital.

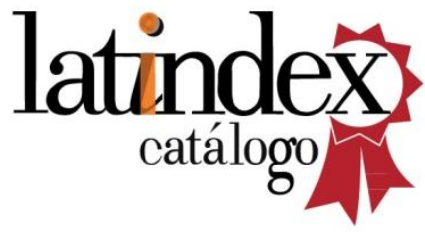

